Published in final edited form as:

Drug Discov Today Dis Mech. 2012 ; 9(1-2): e19-e27. doi:10.1016/j.ddmec.2012.11.003.

\title{
The Role of Androgen Receptor in Breast Cancer
}

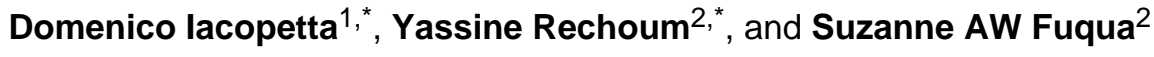 \\ ${ }^{1}$ Department of Pharmaco-Biology, University of Calabria, Arcavacata di Rende, Cosenza, Italy \\ ${ }^{2}$ Baylor College of Medicine, One Baylor Plaza, Houston, Texas 77030, UNITED STATES, \\ 7137981671
}

\begin{abstract}
The androgen receptor (AR) is a proven clinical target in prostate cancer. Recent research indicates that it is an emerging hormonal target in breast cancer as well, with potential clinical benefit in both estrogen receptor(ER) positive and negative tumors. Compared to the ER, AR contains unique functional domains with relevance to its altered role in human breast cancer. The majority of ER-positive tumors express AR, and a significant percentage of ER-negative tumors might benefit from combined targeting of AR and the ErbB2/HER2 oncogene. Signaling downstream of AR might also affect many clinically important pathways which are also emerging clinical targets in breast cancer. AR expression might also play a role during tumor progression to metastatic disease. The role of AR as a new important biomarker in breast cancer will be reviewed herein.
\end{abstract}

\section{Keywords}

androgen receptor; breast cancer; estrogen receptor; clinical target

\section{Introduction}

The androgen receptor (AR) is well-studied in the context of male physiology, and its oncogenic role in the prostate cancer is also well established. As a receptor of precursor ligands of the estrogen receptor (ER), several groups have shed light on the role of the AR in mammary cancer since the mid-1970s. AR is frequently expressed in primary breast tumors; estimated to be between $50 \%$ to $90 \%$ depending on the subtype of breast cancer [1-5]. However, and despite the increasing number of publications, there is still a conundrum whether it acts as a tumor suppressor or an oncogene. In fact, this multifunctional role may be partly explained by the numerous pathways in which AR has been reported to be

\footnotetext{
*Considered Co-First Authors

Publisher's Disclaimer: This is a PDF file of an unedited manuscript that has been accepted for publication. As a service to our customers we are providing this early version of the manuscript. The manuscript will undergo copyediting, typesetting, and review of the resulting proof before it is published in its final citable form. Please note that during the production process errors may be discovered which could affect the content, and all legal disclaimers that apply to the journal pertain.

Conflict of Interest:

The author(s) have no conflict of interest to declare.
} 
involved with, but may also from a metabolic point-of-view, in that androgens are situated between progestin and estrogen synthesis pathways which can have opposing functions.

\section{Androgen Receptor Structure}

The AR is a protein of approximately 918 amino acids in length [6] (Figure 1). It belongs to the steroid nuclear receptor superfamily, a broad family of hormone-regulated transcription factors that share a similar modular tripartite structure including an amino terminus $\left(\mathrm{N}_{\mathrm{t}}\right)$ and a carboxy terminus $\left(\mathrm{C}_{t}\right)$ domain separated by a central domain $[7,8]$. In the genome, AR is represented by a single copy gene, more than $90 \mathrm{~kb}$ in length, located on the X-chromosome at qll-q12 region [9]. It contains 8 exons and its transcript produces an mRNA with an open reading frame (ORF) of about $2.8 \mathrm{~kb}[10,11]$ which encodes two proteins that differ at the $\mathrm{N}_{t}$ with an apparent molecular weight of $87 \mathrm{kDa}$ (the $\mathrm{N}$-truncated form, which translation starts at the internal methionine 188) and the $110 \mathrm{kDafull}-$ length form [12]. The AR protein harbors five functional domains: i) two activation functions AF-1 and AF-2 (also known as transcription-activating functions or TAF); AF-1 is located in the $\mathrm{N}_{\mathrm{t}}$ and represents the major transactivation domain, and AF-2 resides in the $\mathrm{C}_{\mathrm{t}}$ and is highly conserved between species; ii) the DNA binding domain (DBD), necessary for binding to the androgen responsive elements in the regulatory regions of target genes; iii) the ligand-binding domain (LBD), responsible for the binding of agonist and/or antagonist molecules; iv) the nuclear localization function needed for the translocation of AR into the cell nucleus; and finally, v) the hinge domain (HD), located between the DBD and LBD that allows flexibility and plays a role as a binding site for regulatory proteins $[7,13,14]$.

AF-1 and AF-2 are required for the biological functions of nuclear receptors. While AF-1 is ligand-independent, it is constitutively activated with very low conservation across species, AF-2 instead represents the most powerful function; it is ligand-dependent and highly conserved. The AR DBD is also a highly conserved region of approximately 80 amino acids in length, and consists of three a-helices containing two cysteine-rich zinc finger-like modules and a $\mathrm{C}_{\mathrm{t}}$ extension (CTE). The first a helix is involved in DNA sequence recognition and binding. The second and third a-helices arrange to form the second zinc finger module involved in the DNA-dependent dimerization of AR, along with another amino acidic sequence present on the CTE that is important for mediating a specific and high affinity DNA binding [15-17]. After activation by the ligand, AR migrates to the nucleus where it binds to the DNA as a homodimer that recognizes a palindromic consensus sequence 5'-GGTACAnnnTGTTCT-3' that represents the so called androgen response element (ARE). This sequence is common to the consensus glucocorticoid response elements (GRE) [17-19]. Additionally, different selective AREs have been reported to be specifically regulated by AR, but not any other steroid receptor, that we are not detailing here for the sake of brevity but could be reviewed in [20-23], [24], [25] [26] [27-29].

The AR LBD, located on the $\mathrm{C}_{\mathrm{t}}$, partially overlaps AF-2 domain and represents the binding site for androgens and anti-androgens. Androgens are important in normal breast development [30]. In women, they are produced in the ovaries and adrenal glands, and constitute the precursors of estrogens through the estrogen-metabolizing enzymes 
aromatase, steroid sulfatase (STS), estrogen sulfotransferase (EST) and certain types of 17ßhydroxysteroid dehydrogenase $[31,32]$.

During the postmenopausal period, estrogen levels fall, and androgens become the main reservoir to provide estrogen to cells. In postmenopausal breast cancer patients, this alternative metabolic pathway represents the major source of estrogens[33]. The binding of androgens to the LBD causes a conformational change that induces the loss of the heatshock proteins (HSPs) or chaperones, which maintain AR inactive in the cytoplasm after translation. Ligand binding causes a rapid homodimerization and the recruitment of coregulators forming the active AR that migrates to the nucleus, where it recognizes and binds the AREs and regulates gene transcription [34].

The hinge region is a flexible sequence that links the DBD and the LBD in all NRs and allows dimerization and DNA binding. It is poorly conserved and harbors the nuclear localization signal (NLS) necessary for the correct translocation of AR into the nucleus [35]. Adjacent to NLS there are lysine residues that can be modified by acetylation through the transcriptional activators p300/CBP-associated factor, p300, p/CAF and Tip60 (77-80). Mutations in the lysine residues lead to several effects such as impaired ligand-dependent translocation from cytoplasm to nucleus [36]. These mutations do not affect AR transcriptional potential, however. In fact, higher activity is not strictly correlated with the impaired nuclear translocation [62]. As for other nuclear receptors, the AR hinge region is more than a simple flexible linker between the DBD and the LBD but an important regulatory region involved in receptor stability, nuclear localization and intra-nuclear mobility, transactivation activity and DNA binding.

The AR $\mathbf{N}_{\mathbf{t}}$ contains two amino acid stretches polymorphic in length: a glutamine $(\mathrm{Q})$ repeat and glycine $(\mathrm{G})$ repeat (9-36 residues and 10-30 residues respectively). A decrease in the length of Q-repeats enhances transactivation of AR and has been correlated with the increased risk of prostate cancer [37,38]. However, the correlation of the length of the Qrepeats and risk of cancer is controversial in female breast cancer and is thus not a prognostic marker in breast cancer [14,39,40] [41] [42]. The polyG repeat is located between the $\mathrm{N}_{\mathrm{t}}$ transactivation domain and the DBD. It acts as a flexible linker, allowing the $\mathrm{N}_{\mathrm{t}} \mathrm{C}_{\mathrm{t}}$ interactions and seems to be involved in the expression of AR. The effects of the polyG length are not very well understood in female breast cancer.

\section{Genomic and non-genomic signaling}

The AR is reported to exert its effects through three major ways: i) genomic signaling, where AR interacts and directly controls the expression of genes encoded in the DNA; ii) non-genomic signaling, where the nuclear receptor signals via interactions with other proteins, and cross talk between the two signaling mechanisms exists but has yet to be fully understood [43] and; iii) signaling via cross talk with growth factors and cytokines. These combined signaling mechanisms all contribute to AR function in breast cancer. 


\section{Hormonal and non-hormonal mechanisms underlying breast cancer}

Since AR is expressed in $60 \%-70 \%$ of breast cancers, and may play a dual role with ER in both ER-positive cancers, it has drawn the attention of many research groups. ER-/AR+ breast tumors, previously identified as breast tumors with histologically apocrine features (the molecular apocrine subtype), exhibit an intact and active AR signaling [5,44] and therefore have been exploited in AR- targeted therapies. Expression microarray studies [44] have shown the overexpression of steroid-response genes (such as AR and ErbB2) in about $50 \%$ of ER-negative breast cancers. Moreover, cross talk exists between the AR and ErbB2 signaling in molecular apocrine cells, which also involve the epidermal growth factor receptor (EGFR) trafficking signals, has been suggested [45]. Although the exact molecular mechanisms are not clearly understood, it has been shown that AR inhibition, or knockdown, leads to a down-regulation of ERK signaling in tumor xenograft models, and that AR activity was responsible for ErbB2 gene expression. In turn, ERKs are involved in regulation of AR expression; blockade of ERKs phosphorylation results in a decrease of AR expression both in vivo and in vitro in models of molecular apocrine breast cancer.

Additionally, IHC studies have shown an association between AR and the overexpression of ERK targets phospho-Elk-1 and c-Fos, further supporting a fine-tuned regulation between the AR and ERK signaling pathways which involves ErbB2 [45]. An AR-ERK feedback loop using specific AR and MEK inhibitors (flutamide and CI-1040, respectively) has been investigated in molecular apocrine breast cancers [46]. This loop provides an attractive therapeutic target in molecular apocrine breast cancers and also an optional treatment in patients with acquired HER2 targeted therapy resistance [47]. Effectively, androgens (DHT or the synthetic R1881) increase growth responsiveness of MDA-MB-453 human breast cancer cells, which can be blocked by the use of the AR antagonist bicalutamide. This effect is due to the nuclear accumulation and the physical interaction of AR and $\beta$-catenin which, in turn, mediates an up-regulation of HER3 and the activation of HER2/HER3 signaling and downstream effectors PI3K/AKT, responsible for increased cell growth after androgen stimulation [48]. It has been speculated that the loss of ER expression pushes cells to shift from an ER- to an AR-mediated transcriptional priority, but which utilizes similar mechanisms and co-factors as ER and thus promotes cell growth in ER-negative breast cncer. When this happens cell growth becomes refractory to traditional breast cancer therapies (i.e. tamoxifen and aromatase inhibitors treatment) [49] but potentially more sensitive to bicalutamide treatment [48]. Given that AR expression is in part responsible for tumor cell viability and survival, the use of the anti-androgen bicalutamide, alone or in combination with PI3K inhibitors, represents a useful alternative for apocrine breast cancer treatment.

Collins et al. have shown AR expression in relation to tumor stage, pathologic features and molecular phenotype using a large and well-characterized population of women with breast cancer [50]. They demonstrate a better prognosis for women with AR expression in ERpositive tumors [51], while the presence of AR without ER! constitutes the apocrine molecular phenotype (AR+/ER-) characterized by a higher malignancy grade [52]. Few studies have evaluated AR expression in metastatic breast cancers, however one paper has highlighted the relationship between its expression in initial surgically resected metastases 
and end-stage metastases harvested at autopsy and their matched primary breast cancers, using tissues microarray and immunohistochemistry [53]. AR expression in the initial metastasis stage reflects its expression in the corresponding primary tumors, with a trend toward an increased or gained expression in the metastasis. Conversely, in end-stage metastatic breast cancers, AR expression was found to be decreased with a trend toward a complete loss. Although this study has been conducted on a relatively small number of patients, it suggests that the shift of AR expression between the initial and end-stage of metastatic breast cancers (similar to the changes in ER/PR expression) may be responsible for multi-treatment failure, and points out the potential importance of an AR-targeted therapy at an earlier stage before treatment resistance. Thus the study of the AR's role in an experimental system without the potential confounding effects of ERa/PR crosstalk may be helpful [54]. To this purpose, AR has been stably expressed in ERa/PR-negative MCF-10A cells which are non-tumorigenic and a spontaneously immortalized human breast epithelial cell line. The characterization of this cell model showed AR signaling which can lead to increased gene transcription via AREs and increased mitogen-activated protein kinase (MAPK) signaling. Additionally, AR signaling was mediated through the cyclin-dependent kinase (CDK) inhibitor p21 (the promoter contains an ARE [55]). Co-stimulation of EGFR signaling and AR led to a growth-inhibitory effect due to hyperactivation of MAPK pathway, whereas stimulation by either EGFR or AR alone resulted in cellular proliferation. These findings may offer some explanation for the paradoxical growth responses to AR ligand binding in different human breast cancer cell lines, and also suggest that p21 may be important for mediating AR signaling in human breast epithelial cells. Thus, it can be hypothesized that $\mathrm{p} 21$ may represent a prognostic factor for sensitivity versus resistance to AR-directed therapies. The expression of AR in triple negative breast tumors (ER/PR/ HER2-negative) suggests that it can function independently of ER and PR, and act in concert with other signal transduction pathways producing enhanced cell growth and an aggressive phenotype. Graham et al [56] showed that Zinc-finger enhancer binding protein (ZEB1), a transcription factor involved in the progression of cancer, up-regulates AR expression and its signaling in triple negative breast cancer cell lines. However, it produces opposite effects in breast cancer cell lines expressing ER. Moreover AR signaling blockade with bicalutamide suppressed ZEB1 expression, resulting in morphologic changes, AR mRNA and protein reduction, and a higher sensitivity to anti-androgens. Thus, the cross-talk between AR and ZEB1 appears to be a critical pathway in cancer progression and, at the same time, a strong predictor of poor patient survival and metastasis.

Many immunohistochemistry studies correlate the decrease of AR expression with cancer progression, and androgens such as fluoxymesterone have been used as second-line hormonal therapy for advanced breast cancers. The recent discovery that the tumor suppressor PTEN cell-signaling pathway, involved in cell proliferation, migration and apoptosis, may be directly regulated by AR also helps shed light on the opposing effects of AR in prostate and some breast cancers [57]. In fact, androgens stimulate PTEN transcriptional expression, through $\mathrm{AR}$, in some breast cancer cells, and the changes in the androgenic hormonal milieu, which occur in the menopausal state, may modulate PTEN expression. More recently it has been shown that this signal transduction pathway is a complicated network, containing multiple levels of feedback. In fact, AKT and PTEN 
negatively regulate each other and this effect is mediated by the transcriptional factor GATA2, which suppresses PTEN transcription in both an AR-dependent and independent manner [58]. A comparison between expression gene levels was conducted by Singh et al. [59] in order to elucidate the functional role of AR, growth factor receptor genes (EGFR, ERBB2, ERBB3), and the multidrug resistant gene MDR1 and how these molecular biomarkers can be used to predict therapy response. Elevated levels of AR expression have been found before therapy (together with EGFR, ERBB2 and MDR1 in responder patients), but these levels are reduced after treatment in patients who respond to neoadjuvant chemotherapy. Conversely, in patients whom do not respond to neoadjuvent chemotherapy, AR and ERBB3 expression appears to be up-regulated, confirming AR regulation by ERBB3. Thus, AR seems to be regulated by this chemo-treatment and may be predictive of the success in individual cancer patients with locally advanced breast carcinoma. It is indicated that AR expression is a common feature of BRCA2 positive and BRCA1/2negative breast cancers with a positive family history (70\% of AR positive staining), whereas the majority of BRCA1-related tumors are AR-negative. This suggests that the biological phenotype of BRCA1 mutated tumors differs from that of BRCA2-related tumors and seems to be hormone-independent although AR expression is dependent on well-known prognostic/predictive factors (grade, receptorial state). Additionally, $20 \%$ of hereditary BRCA1-and BRCA2-related breast carcinomas have been found to be triple-negative but AR positive, which might represent an optional approach to consider in a hormonal therapy treatment. However, BRCA1/2 mutation increases breast cancer risk throughout a woman's lifetime and could exert a cooperative negative effect in postmenopausal women in which the circulating androgen levels increase. In fact, Park et al demonstrated that BRCA1 is a coactivator of AR and can directly modulate its signaling [60]. Almost half of BRCA-related tumors of postmenopausal women have been found positive for AR expression in these studies [61].

\section{AR as a predictive/prognostic marker}

$\mathrm{Hu}$ et al. have conducted one of the few large studies examining the association between AR expression and breast cancer [62]. They show that the association of AR status and breast cancer survival is dependent on ER expression, with significantly improved survival in ERpositive tumors. AR expression was associated with favorable clinic-pathological parameters and better outcomes in ER-positive breast cancers. AR expression levels were significantly higher in endocrine responsive tumors, including non-TNBC, and in about a quarter of patients with ER-negative tumors. AR was found important for survival outcome and its prognostic significance was demonstrated in multivariate analyses for disease free survival (DFS) but not overall survival (OS). A role in prediction of systemic treatments has been suggested, given that about $90 \%$ of ER-positive patients received endocrine or chemoendocrine therapy. In the ER-negative and TNBC subsets there was statistically no prognostic impact of AR and ER-negative tumor patients did not benefit from endocrine therapy, showing a poorer prognosis with respect to ER-positive tumor patients. However, a_statistically significant association between AR expression and HER2 positivity was demonstrated in molecular apocrine tumors, in addition to a borderline significantly poorer OS, supporting the idea that the blockade of both AR and HER2 pathways might be used in therapy [63]. Another large study was conducted on 980 Chinese patients with invasive 
breast carcinoma which showed AR to be more frequently expressed than ER and PR, and its expression was closely associated with ER and PR. It was higher in ER+ subtypes than in ER- subtypes, and was associated with more favorable pathologic features, including small tumor size and lower Ki-67 expression, a marker of proliferation in breast cancer [64]. A retrospective study comprising 287 female patients with TNBC diagnosed without any evidence of distant metastasis at the time of surgery revealed the presence of AR in $25.8 \%$ of TNBC, lower than in ER-positive breast cancers. AR-negative patients have a higher proportion of positive lymph node and poor prognosis, whereas AR-positivity is significantly associated with a longer DFS and OS [65]. AR expression by immunohistochemistry has been evaluated on a tissue microarray of 673 core biopsies from primary breast cancer patients treated with neoadjuvant docetaxel/doxorubicin/ cyclophosphamide (TAC) chemotherapy in the prospective GeparTrio phase-III trial in order to determine the predictive impact of $\mathrm{AR}$ on the rate of pathological complete response (pCR) and its potential role as prognostic marker, particularly in TNBC patients and in patients not achieving a pCR [66]. It has been demonstrated that AR negativity is predictive for pathological response (pCR) to chemotherapy and suggested additional treatments such as bicalutamide or abiraterone acetate for TNBC/AR-positive patients achieving no $\mathrm{pCR}$,

\section{Methods for detection of AR expression in breast tumors}

Several methods have been used to evaluate AR expression in breast cancer samples. One of the most used is immunohistochemistry (IHC) using specific antibodies directed against AR in whole sections (WS) and/or in tissue microarrays (TMA) [67,68]. Using TMA, Niemeier et al, showed that AR is expressed in almost all ER-positive tumors, however, in ERnegative tumors AR expression was predominantly seen in the HER2 molecular tumor subgroup. Additionally, in some cases, AR was detected in the TN tumors, depending on the apocrine differentiation state [67]. The IHC method also allows a tissue specific determination of AR and the discrimination between the nuclear and cytoplasmic pools even in small sample sizes. The "charcoal method", which offers a valid alternative to check for AR expression in breast cancer cells, uses a labeled AR ligand [69]. However, with respect to the IHC techniques, the charcoal method is not very well supported by large studies.

A third technique adopted for AR detection, but also for other NRs, is an RNA-based method starting from archived breast tissue sections embedded in paraffin and fixed with formalin on slides [70]. The reverse phase protein lysate array is a different approach for AR detection introduced by Gonzalez-Angulo et al. This technique allows the examination of AR levels in breast cancer tumor subtypes and the impact of AR expression on relapse-free survival (RFS) and overall survival in breast cancer patients [2]. The reverse phase protein lysate array method provides an absolute quantification of AR contrary to other techniques that simply classify tumor samples as "AR-positive or negative". However, one of its limitations is the possible contamination of the tumor sample with stroma. In addition to these techniques in which frozen or paraffin embedded samples from tumors are used, the Positron Emission Tomography (PET) method represents a non-invasive and alternative nuclear imaging technique that allows the visualization and quantification, in vivo, hormone receptor expression throughout the entire body using a tracer in a single session [71]. 


\section{Prognostic relevance in clinical progression}

The AR is frequently expressed in many types of breast carcinoma, including both invasive and in situ ductal carcinomas (IDC and DCIS respectively) [1], lobular carcinoma [72,73], BRCA-mutated tumors [73], and mammary Paget's disease [74]. Its expression is correlated with a better prognosis for breast carcinoma. A univariate analysis showed that AR has prognostic value in primary breast cancer, along with the classical histological and cell biological parameters which are ER! status, tumor size, tumor grade, lymph node status, and high level of Ki-67 [75]. More recently it has been demonstrated that AR overexpression may confer tamoxifen (Tam) resistance in breast cancer cells through activation of cell signaling pathways that facilitate ERa activation. It is likely that AR and ERa interact in the presence of Tam, forming a complex that is recruited to ER-responsive gene promoters [76]. Thus, it seems that AR is not only frequently expressed in breast carcinoma and related to prognosis, but also may serve as a predictive marker for hormone therapy and a potential therapeutic target for breast cancer, especially for ER/PR-negative breast cancer. However, the relationship between AR expression, tumor progression and grade is not clearly defined. Hanley et al explored the expression of AR in breast cancer and its relationship with tumor progression (e.g. DCIS vs. IDC), tumor cell nuclear grades (high grade [HG] vs. non-high grade [NHG]) and expression of ER, PR, and HER-2 [77]. They found AR expression both in situ (89\%) and in invasive NHG tumors (92\%) suggesting that AR, along with ER and PR, could be important for early carcinogenesis. In HG tumors, HG-DCIS and HG-IDC differ in AR expression (93\% and 55\%, respectively) and have a higher AR expression with respect to ER and PR. This suggests that the lack of AR expression could play a major role in the transformation of HG-DCIS to HG-IDC. AR may represent a potential therapeutic target for HG carcinomas in AR-positive/HER-2-negative tumors, The difference in expression patterns among AR, ER, PR, and HER-2 is very marked in HG but not in NHG carcinomas and the significant loss of AR expression in HG-IDC (55\%) with respect to HGDCIS (93\%) suggests a possible role in the invasive transformation of HG carcinomas. HER-2 status also influences the relevance of AR as prognostic factor, since AR positivity is insufficient to compensate the unfavorable effect of HER2/neu overexpression on the patient's prognosis [78].

Peters et al. showed that AR levels are significantly associated with disease outcome in ERa-positive breast cancer, a 3.0-fold increased risk of relapse and a 4.6-fold increased risk of cancer-related death for patients with lower than median percent AR positivity, and that AR is a direct repressor of ERa signaling in breast cancer cells [79]. These findings suggest that, at least in ERa-positive breast cancer, AR is an independent predictor of outcome. In agreement with these results, a recent study from Castellano et al. showed that AR expression is an independent prognostic factor of better outcome in patients with ERpositive breast cancers, in particular for the subset of luminal type B tumors [80]. In the group of patients treated with endocrine therapy associated with chemotherapy, AR positivity represents a statistically strong prognostic factor for both time to relapse (TTR) and disease specific survival (DSS). Moreover, according to the different chemotherapeutic regimens, AR expression reveals itself as a marker of good prognosis in patients treated with cyclophosphamide, methotrexate, and fluorouracil (CMF) and anthracycline. The authors 
confirmed that AR status is an independent prognostic factor in ERa-positive breast cancers and in patients treated with endocrine therapy and chemotherapy for both TTR and DSS, and only for the TTR in patients treated with endocrine therapy alone.

\section{Role in the prediction of response to hormonal therapy}

Hormone therapy is one of the most used therapeutic modalities in the treatment of breast cancer, given that about $70 \%$ of breast cancers express ER, PR or both and they are considered sensitive to endocrine treatment with a favorable benefit-to-toxicity ratio. The most frequently used drugs in endocrine therapy are tamoxifen (a selective estrogen receptor modulator or SERM), Fulvestrant (a pure ERa antagonist) and the AIs (estrogen synthesis inhibitors which block aromatase) [71]. A new steroid biosynthesis inhibitor is the abiraterone acetate which blocks CYP-17A1 potently and specifically, preventing the conversion of androgen precursors to androgens and consequently estrogens. This drug is in phase I/II clinical trials in postmenopausal women with advanced or metastatic breast cancer. For the portion of patients whose tumors belong to the HER2-positive subtype $(15 \%)$, trastuzumab (a monoclonal antibody) is often used in combination with chemotherapy [81-83]. A new option of treatment under phase II clinical investigation, TDM1, combines the anti-HER2 monoclonal antibody, trastuzumab, and the anti-microtubule chemotherapy agent maytansine (DM1). This combination allows targeted delivery of a strong cytotoxic agent to tumor cells overexpressing HER2 [84] which might be appropriate for AR/HER2+ patients.

However, chemotherapy and radiotherapy represent thus far the exclusive option for the treatment of TN breast tumors because of the lack of a nuclear receptor target. AR is expressed in almost $70 \%$ of these breast tumors and therefore, could be used as a target in these subtypes. Androgen therapy has been occasionally given to ER-positive patients after all other hormone therapies have failed, and have a comparable efficacy with that of current hormonal treatments for advanced breast cancer, but its use declined with the advent of Tam and AIs $[4,85]$. Dehydroepiandosterone sulfate (DHEAS) has been used in combination with the third generation AIs in order to prevent the conversion of DHEAS to estrone and estradiol, and may represent an effective treatment for the apocrine subtype breast cancer [86]. For instance, synthetic progestins such as medroxyprogesterone acetate (MPA) were in use since the 1950s for metastatic breast cancer, but their use declined following the advent of Tam and AIs. More recently, there is a renewed interest in MPA as an alternative hormonal treatment for early disease and in the advanced setting when conventional therapies fail. Synthetic progestins often bind with high specificity to other steroid receptors including those for the androgen and glucocorticoid receptors, thus the androgenic side effects observed in women taking MPA suggest that the AR may be implicated in its activity in vivo $[4,11]$. Clinical data suggested that $A R$, but not $P R$, is directly involved in progestininduced regression of tumor growth, acting as a receptor for MPA, and that sensitivity of mammary cancers is seen only at high progestin concentrations [87,88]. Furthermore, MPA inhibits proliferation of AR-positive but not AR-negative breast cancer cell lines, and this inhibition can be reversed by co-treatment with specific AR antagonists, demonstrating the unequivocal role of AR. The importance of AR in predicting response to MPA is still underlined by the conservation of its structure or interactions. In fact, mutations or structural 
alterations lead to a switch of MPA characteristics from an agonist to antagonist of AR, thus a poorer or null response to progestin therapy, even though AR still retains its ability to bind androgens. These data from Buchanan et al [4] strongly support the protective role of AR and that the effects of androgens on tumor growth are specifically mediated through AR activation rather than estrogen signaling. Moreover, a balance between estrogen and androgen signaling may explain the in vivo effects of androgens on mammary epithelial cell growth, and the link of AR with breast cancer risk in women treated with MPA as a component of hormonal therapy [89].

Aromatase inhibitors can be classified either as steroidal (type I inhibitors) or nonsteroidal (type II inhibitors), based on their chemical structure. For instance, anastrozole and letrozole are nonsteroidal, whereas exemestane is a steroidal inhibitor. These drugs show classindependent differences in terms of potency or class-specific differences in terms of their mechanism of binding to aromatase and clinical relevance [85]. The research group of Dr. Brodie has extensively studied the effects of AIs on breast cancer and have shown that the antiproliferative effect of androgens on breast cancer cells is mediated by AR. Using the AR inhibitor bicalutamide, they suggested that the letrozole's antiproliferative mechanism of action may be due not only to the reduction in estrogen biosynthesis, but also to the inhibitory effects of androgens acting via the AR [90,91]. The steroidal inhibitor exemestane was shown not to affect AR expression levels. However, high phosphorylation levels of Akt Ser473 were detected, leading to the conclusion that inhibition of aromatase activity and estrogen production by exemestane affects molecules that are upstream of ER signaling pathways, and may be useful to identify patients responsive to this drug [92]. Since AR has been shown to be expressed in breast cell lines, like MCF-7, and is an important regulatory factor, the question of AR being involved in Tam resistance became an interesting field of study. Previous data from Khalkhali-Ellis et al, showed that Tam induces the tumor suppressor gene maspin in MCF-7 cells. Moreover, the non-steroidal antiandrogen, flutamide, seems also to induce the maspin promoter which may be mediated via AR, suggesting its negative involvement in cancer progression and the use of alternative approaches such as combined protocols to block both ER and AR in breast cancer [93]. A clinical study using flutamide in postmenopausal women indicated that it cannot be used by itself as a valid therapy for postmenopausal patients, but may improve the efficacy of other hormonal agents [94]. Thus, a combined endocrine therapy, effective not only on ERpositive but also on AR-positive cells, may represent a way to delay the appearance of clinical resistance. For instance, Di Monaco et al showed that the combined use of Tam and hydroxyflutamide (OH-flutamide), the biological active flutamide metabolite, is additive and exerts an antiproliferative action on breast cancer cells [95]. Studies supporting the demonstration that AR levels could affect the response to Tam in ERa-positive breast cancer cells have been clearly shown by De Amicis et al. The observation that Tam shows an agonist activity on ERa at ERE sites, which can be blocked by treatment with bicalutamide provides evidence that ERa and AR may interact in vivo, and that their cooperation on genomic targets may a factor underlying breast tumor progression and hormonal resistance [76]. In brief, all these data clearly indicate the role of $A R$ as a predictor of the hormonal response in breast cancer treatment. New understanding of the AR gene, gene transcripts and variants, post-translational modifications of the receptor protein and interactions with other 
signaling networks in tumor cells are needed to provide insight into predicting the response to therapy.

\section{Clinical hormonal target}

Despite the abundance of data in literature regarding the use of drugs targeting AR in vitro and in vivo, it is not uncommon that we observe different effects in clinical studies. For instance, flutamide exhibits antiproliferative actions on breast cancer in vitro and in animal experiments. However, in a phase II clinical trial, flutamide alone did not show a favorable response in postmenopausal breast cancer women [94]. Recent in vitro and in vivo data show that AR expression is elevated in Tam-resistant breast cancer samples, and that AR could assist Tam-dependent stimulation of ERa action leading to a cell proliferation [76]. This clearly raises the question whether a blockade of AR may be an effective and new target to overcome hormone resistance. In this setting, flutamide may also play an important role in ccombined hormonal-chemotherapy treatments. Active research in this area is currently underway, and it is predicted that AR will prove to be a useful target, especially in patients with hormone resistant disease, such as those patients with ER-positive metastatic disease or tumors of the TN subtype.

\section{REFERENCES}

1. Moinfar F, et al. Androgen receptors frequently are expressed in breast carcinomas: potential relevance to new therapeutic strategies. Cancer. 2003; 98(4):703-711. [PubMed: 12910513]

2. Gonzalez-Angulo AM, et al. Androgen receptor levels and association with PIK3CA mutations and prognosis in breast cancer. Clin Cancer Res. 2009; 15(7):2472-2478. [PubMed: 19276248]

3. Birrell SN, et al. Androgens induce divergent proliferative responses in human breast cancer cell lines. J Steroid Biochem Mol Biol. 1995; 52(5):459-467. [PubMed: 7748811]

4. Buchanan G, et al. Decreased androgen receptor levels and receptor function in breast cancer contribute to the failure of response to medroxyprogesterone acetate. Cancer Res. 2005; 65(18): 8487-8496. [PubMed: 16166329]

5. Doane AS, et al. An estrogen receptor-negative breast cancer subset characterized by a hormonally regulated transcriptional program and response to androgen. Oncogene. 2006; 25(28):3994-4008. [PubMed: 16491124]

6. Chang CS, et al. Structural analysis of complementary DNA and amino acid sequences of human and rat androgen receptors. Proc Natl Acad Sci U S A. 1988; 85(19):7211-7215. [PubMed: 3174628]

7. Knudsen KE, Scher HI. Starving the addiction: new opportunities for durable suppression of AR signaling in prostate cancer. Clin Cancer Res. 2009; 15(15):4792-4798. [PubMed: 19638458]

8. Callewaert L, et al. Interplay between two hormone-independent activation domains in the androgen receptor. Cancer Res. 2006; 66(1):543-553. [PubMed: 16397271]

9. Brown CJ, et al. Androgen receptor locus on the human X chromosome: regional localization to Xq11-12 and description of a DNA polymorphism. Am J Hum Genet. 1989; 44(2):264-269. [PubMed: 2563196]

10. Roy AK, et al. Androgen receptor: structural domains and functional dynamics after ligandreceptor interaction. Ann N Y Acad Sci. 2001; 949:44-57. [PubMed: 11795379]

11. Gelmann EP. Molecular biology of the androgen receptor. J Clin Oncol. 2002; 20(13):3001-3015. [PubMed: 12089231]

12. Wilson CM, McPhaul MJ. A and B forms of the androgen receptor are present in human genital skin fibroblasts. Proc Natl Acad Sci U S A. 1994; 91(4):1234-1238. [PubMed: 8108393]

13. Centenera MM, et al. The contribution of different androgen receptor domains to receptor dimerization and signaling. Mol Endocrinol. 2008; 22(11):2373-2382. [PubMed: 18617596] 
14. Nicolas Diaz-Chico B, et al. Androgens and androgen receptors in breast cancer. J Steroid Biochem Mol Biol. 2007; 105(1-5):1-15. [PubMed: 17631997]

15. Luisi BF, et al. Crystallographic analysis of the interaction of the glucocorticoid receptor with DNA. Nature. 1991; 352(6335):497-505. [PubMed: 1865905]

16. Haelens A, et al. Androgen-receptor-specific DNA binding to an element in the first exon of the human secretory component gene. Biochem J. 2001; 353(Pt 3):611-620. [PubMed: 11171058]

17. Claessens F, et al. Selective DNA binding by the androgen receptor as a mechanism for hormonespecific gene regulation. J Steroid Biochem Mol Biol. 2001; 76(1-5):23-30. [PubMed: 11384860]

18. Cato AC, et al. The hormone response element of the mouse mammary tumour virus DNA mediates the progestin and androgen induction of transcription in the proviral long terminal repeat region. EMBO J. 1987; 6(2):363-368. [PubMed: 3034586]

19. Ham J, et al. Characterization of response elements for androgens, glucocorticoids and progestins in mouse mammary tumour virus. Nucleic Acids Res. 1988; 16(12):5263-5276. [PubMed: 2838812]

20. Claessens F, et al. The androgen-specific probasin response element 2 interacts differentially with androgen and glucocorticoid receptors. J Biol Chem. 1996; 271(32):19013-19016. [PubMed: 8702568]

21. Schoenmakers E, et al. Differential DNA binding by the androgen and glucocorticoid receptors involves the second $\mathrm{Zn}$-finger and a C-terminal extension of the DNA-binding domains. Biochem J. 1999; 341(Pt 3):515-521. [PubMed: 10417312]

22. Verrijdt G, et al. Change of specificity mutations in androgen-selective enhancers. Evidence for a role of differential DNA binding by the androgen receptor. J Biol Chem. 2000; 275(16):1229812305. [PubMed: 10766869]

23. Verrijdt G, et al. Functional interplay between two response elements with distinct binding characteristics dictates androgen specificity of the mouse sex-limited protein enhancer. J Biol Chem. 2002; 277(38):35191-35201. [PubMed: 12107189]

24. Verrijdt G, et al. Androgen specificity of a response unit upstream of the human secretory component gene is mediated by differential receptor binding to an essential androgen response element. Mol Endocrinol. 1999; 13(9):1558-1570. [PubMed: 10478846]

25. Barbulescu K, et al. New androgen response elements in the murine pem promoter mediate selective transactivation. Mol Endocrinol. 2001; 15(10):1803-1816. [PubMed: 11579212]

26. Steketee K, et al. A bioinformatics-based functional analysis shows that the specifically androgenregulated gene SARG contains an active direct repeat androgen response element in the first intron. J Mol Endocrinol. 2004; 33(2):477-491. [PubMed: 15525603]

27. Bolton EC, et al. Cell- and gene-specific regulation of primary target genes by the androgen receptor. Genes Dev. 2007; 21(16):2005-2017. [PubMed: 17699749]

28. Massie CE, et al. New androgen receptor genomic targets show an interaction with the ETS1 transcription factor. EMBO Rep. 2007; 8(9):871-878. [PubMed: 17721441]

29. Wang Q, et al. A hierarchical network of transcription factors governs androgen receptordependent prostate cancer growth. Mol Cell. 2007; 27(3):380-392. [PubMed: 17679089]

30. McPhaul MJ, et al. Androgen resistance caused by mutations in the androgen receptor gene. FASEB J. 1991; 5(14):2910-2915. [PubMed: 1752359]

31. Honma N, et al. Estrogen-metabolizing enzymes in breast cancers from women over the age of 80 years. J Clin Endocrinol Metab. 2006; 91(2):607-613. [PubMed: 16303840]

32. Pasqualini JR, Chetrite GS. Recent insight on the control of enzymes involved in estrogen formation and transformation in human breast cancer. J Steroid Biochem Mol Biol. 2005; 93(2-5): 221-236. [PubMed: 15860265]

33. Chetrite GS, et al. Comparison of estrogen concentrations, estrone sulfatase and aromatase activities in normal, and in cancerous, human breast tissues. J Steroid Biochem Mol Biol. 2000; 72(1-2):23-27. [PubMed: 10731634]

34. Agoulnik IU, Weigel NL. Androgen receptor action in hormone-dependent and recurrent prostate cancer. J Cell Biochem. 2006; 99(2):362-372. [PubMed: 16619264] 
35. Zhou ZX, et al. A ligand-dependent bipartite nuclear targeting signal in the human androgen receptor. Requirement for the DNA-binding domain and modulation by NH2-terminal and carboxyl-terminal sequences. J Biol Chem. 1994; 269(18):13115-13123. [PubMed: 8175737]

36. Thomas M, et al. Androgen receptor acetylation site mutations cause trafficking defects, misfolding, and aggregation similar to expanded glutamine tracts. J Biol Chem. 2004; 279(9): 8389-8395. [PubMed: 14670946]

37. Mononen N, et al. Androgen receptor CAG polymorphism and prostate cancer risk. Hum Genet. 2002; 111(2):166-171. [PubMed: 12189490]

38. Harada $\mathrm{N}$, et al. Involvement of three glutamine tracts in human androgen receptor transactivation. J Steroid Biochem Mol Biol. 118(1-2):77-84. [PubMed: 19833203]

39. $\mathrm{Yu} \mathrm{H}$, et al. Shorter $\mathrm{CAG}$ repeat length in the androgen receptor gene is associated with more aggressive forms of breast cancer. Breast Cancer Res Treat. 2000; 59(2):153-161. [PubMed: 10817350]

40. Elhaji YA, et al. The polymorphic CAG repeat of the androgen receptor gene: a potential role in breast cancer in women over 40. Breast Cancer Res Treat. 2001; 70(2):109-116. [PubMed: 11768600]

41. Iobagiu C, et al. Microsatellite profile in hormonal receptor genes associated with breast cancer. Breast Cancer Res Treat. 2006; 95(2):153-159. [PubMed: 16317584]

42. Wang W, et al. Androgen receptor and prostate-specific antigen gene polymorphisms and breast cancer in African-American women. Cancer Epidemiol Biomarkers Prev. 2005; 14(12):29902994. [PubMed: 16365023]

43. Rahman F, Christian HC. Non-classical actions of testosterone: an update. Trends Endocrinol Metab. 2007; 18(10):371-378. [PubMed: 17997105]

44. Farmer P, et al. Identification of molecular apocrine breast tumours by microarray analysis. Oncogene. 2005; 24(29):4660-4671. [PubMed: 15897907]

45. Chia KM, et al. A feedback loop between androgen receptor and ERK signaling in estrogen receptor-negative breast cancer. Neoplasia. 2011; 13(2):154-166. [PubMed: 21403841]

46. Naderi A, et al. Synergy between inhibitors of androgen receptor and MEK has therapeutic implications in estrogen receptor-negative breast cancer. Breast Cancer Res. 2011; 13(2):R36. [PubMed: 21457548]

47. Ni M, et al. Targeting androgen receptor in estrogen receptor-negative breast cancer. Cancer cell. 2011; 20(1):119-131. [PubMed: 21741601]

48. Lehmann BD, et al. Identification of human triple-negative breast cancer subtypes and preclinical models for selection of targeted therapies. J Clin Invest. 2011; 121(7):2750-2767. [PubMed: 21633166]

49. Robinson JL, et al. Androgen receptor driven transcription in molecular apocrine breast cancer is mediated by FoxA1. EMBO J. 2011; 30(15):3019-3027. [PubMed: 21701558]

50. Collins LC, et al. Androgen receptor expression in breast cancer in relation to molecular phenotype: results from the Nurses' Health Study. Mod Pathol. 2011; 24(7):924-931. [PubMed: 21552212]

51. Garreau JR, et al. Transgenic introduction of androgen receptor into estrogen-receptor-, progesterone-receptor-, and androgen-receptor-negative breast cancer cells renders them responsive to hormonal manipulation. Am J Surg. 2006; 191(5):576-580. [PubMed: 16647340]

52. Lin Fde M, et al. Coordinated expression of oestrogen and androgen receptors in HER2-positive breast carcinomas: impact on proliferative activity. J Clin Pathol. 2012; 65(1):64-68. [PubMed: 22039288]

53. Cimino-Mathews A, et al. Androgen receptor expression is usually maintained in initial surgically resected breast cancer metastases but is often lost in end-stage metastases found at autopsy. Hum Pathol. 2011

54. Garay JP, et al. The growth response to androgen receptor signaling in ERalpha-negative human breast cells is dependent on p21 and mediated by MAPK activation. Breast Cancer Res. 2012; 14(1):R27. [PubMed: 22321971] 
55. Lu S, et al. Androgen regulation of the cyclin-dependent kinase inhibitor p21 gene through an androgen response element in the proximal promoter. Mol Endocrinol. 1999; 13(3):376-384. [PubMed: 10076995]

56. Graham TR, et al. Reciprocal regulation of ZEB1 and AR in triple negative breast cancer cells. Breast Cancer Res Treat. 2010; 123(1):139-147. [PubMed: 19921427]

57. Wang Y, et al. Differential regulation of PTEN expression by androgen receptor in prostate and breast cancers. Oncogene. 2011; 30(42):4327-4338. [PubMed: 21532617]

58. Wang Y, et al. GATA2 negatively regulates PTEN by preventing nuclear translocation of androgen receptor and by androgen-independent suppression of PTEN transcription in breast cancer. Hum Mol Genet. 2012; 21(3):569-576. [PubMed: 22021428]

59. Singh LC, et al. Study on predictive role of AR and EGFR family genes with response to neoadjuvant chemotherapy in locally advanced breast cancer in Indian women. Med Oncol. 2011

60. Park JJ, et al. Breast cancer susceptibility gene 1 (BRCAI) is a coactivator of the androgen receptor. Cancer Res. 2000; 60(21):5946-5949. [PubMed: 11085509]

61. Pristauz G, et al. Androgen receptor expression in breast cancer patients tested for BRCA1 and BRCA2 mutations. Histopathology. 2010; 57(6):877-884. [PubMed: 21166701]

62. Hu R, et al. Androgen receptor expression and breast cancer survival in postmenopausal women. Clin Cancer Res. 2011; 17(7):1867-1874. [PubMed: 21325075]

63. Park S, et al. Androgen receptor expression is significantly associated with better outcomes in estrogen receptor-positive breast cancers. Ann Oncol. 2011; 22(8):1755-1762. [PubMed: 21310761]

64. Qi JP, et al. Expression of the androgen receptor and its correlation with molecular subtypes in 980 chinese breast cancer patients. Breast Cancer (Auckl). 2012; 6:1-8. [PubMed: 22259247]

65 . He J, et al. Prognostic value of androgen receptor expression in operable triple-negative breast cancer: a retrospective analysis based on a tissue microarray. Med Oncol. 2011

66. Loibl S, et al. Androgen receptor expression in primary breast cancer and its predictive and prognostic value in patients treated with neoadjuvant chemotherapy. Breast Cancer Res Treat. 2011; 130(2):477-487. [PubMed: 21837479]

67. Niemeier LA, et al. Androgen receptor in breast cancer: expression in estrogen receptor-positive tumors and in estrogen receptor-negative tumors with apocrine differentiation. Mod Pathol. 2010; 23(2):205-212. [PubMed: 19898421]

68. Soiland $\mathrm{H}$, et al. Prognostic relevance of androgen receptor detection in operable breast cancer. $\mathrm{J}$ Surg Oncol. 2008; 98(7):551-558. [PubMed: 18937259]

69. Lea OA, et al. Improved measurement of androgen receptors in human breast cancer. Cancer Res. 1989; 49(24 Pt 1):7162-7167. [PubMed: 2582456]

70. Smith RA, et al. Detection of mRNA levels for the estrogen alpha, estrogen beta and androgen nuclear receptor genes in archival breast cancer tissue. Cancer Lett. 2006; 237(2):248-255. [PubMed: 16046054]

71. Hospers GA, et al. PET imaging of steroid receptor expression in breast and prostate cancer. Curr Pharm Des. 2008; 14(28):3020-3032. [PubMed: 18991716]

72. Riva C, et al. Immunohistochemical study of androgen receptors in breast carcinoma. Evidence of their frequent expression in lobular carcinoma. Virchows Arch. 2005; 447(4):695-700. [PubMed: 16075292]

73. Berns EM, et al. Androgen pathway dysregulation in BRCA1-mutated breast tumors. Breast Cancer Res Treat. 2003; 79(1):121-127. [PubMed: 12779088]

74. Liegl B, et al. Androgen receptors are frequently expressed in mammary and extramammary Paget's disease. Mod Pathol. 2005; 18(10):1283-1288. [PubMed: 15920545]

75. Kuenen-Boumeester V, et al. The clinical significance of androgen receptors in breast cancer and their relation to histological and cell biological parameters. Eur J Cancer. 1996; 32A(9):1560 1565. [PubMed: 8911118]

76. De Amicis F, et al. Androgen receptor overexpression induces tamoxifen resistance in human breast cancer cells. Breast Cancer Res Treat. 2010; 121(1):1-11. [PubMed: 19533338] 
77. Hanley K, et al. Lack of expression of androgen receptor may play a critical role in transformation from in situ to invasive basal subtype of high-grade ductal carcinoma of the breast. Hum Pathol. 2008; 39(3):386-392. [PubMed: 18187183]

78. Schippinger W, et al. Evaluation of the prognostic significance of androgen receptor expression in metastatic breast cancer. Virchows Arch. 2006; 449(1):24-30. [PubMed: 16628414]

79. Peters AA, et al. Androgen receptor inhibits estrogen receptor-alpha activity and is prognostic in breast cancer. Cancer Res. 2009; 69(15):6131-6140. [PubMed: 19638585]

80. Castellano I, et al. Androgen receptor expression is a significant prognostic factor in estrogen receptor positive breast cancers. Breast Cancer Res Treat. 2010

81. Lohrisch C, Piccart M. HER2/neu as a predictive factor in breast cancer. Clin Breast Cancer. 2001; 2(2):129-135. discussion 136-127. [PubMed: 11899784]

82. Rakha EA, et al. Prognostic markers in triple-negative breast cancer. Cancer. 2007; 109(1):25-32. [PubMed: 17146782]

83. Goldhirsch A, et al. Thresholds for therapies: highlights of the St Gallen International Expert Consensus on the primary therapy of early breast cancer 2009. Ann Oncol. 2009; 20(8):13191329. [PubMed: 19535820]

84. Lewis Phillips GD, et al. Targeting HER2-positive breast cancer with trastuzumab-DM1, an antibody-cytotoxic drug conjugate. Cancer research. 2008; 68(22):9280-9290. [PubMed: 19010901]

85. Miller WR, et al. Aromatase inhibitors: are there differences between steroidal and nonsteroidal aromatase inhibitors and do they matter? Oncologist. 2008; 13(8):829-837. [PubMed: 18695261]

86. Hardin C, et al. A new hormonal therapy for estrogen receptor-negative breast cancer. World J Surg. 2007; 31(5):1041-1046. [PubMed: 17429562]

87. Birrell SN, et al. Medroxyprogesterone acetate therapy in advanced breast cancer: the predictive value of androgen receptor expression. J Clin Oncol. 1995; 13(7):1572-1577. [PubMed: 7602345]

88. Teulings FA, et al. Estrogen, androgen, glucocorticoid, and progesterone receptors in progestininduced regression of human breast cancer. Cancer Res. 1980; 40(7):2557-2561. [PubMed: 6248208]

89. Birrell SN, et al. Role of the androgen receptor in human breast cancer. J Mammary Gland Biol Neoplasia. 1998; 3(1):95-103. [PubMed: 10819508]

90. Labrie F, et al. Endocrine and intracrine sources of androgens in women: inhibition of breast cancer and other roles of androgens and their precursor dehydroepiandrosterone. Endocr Rev. 2003; 24(2):152-182. [PubMed: 12700178]

91. Macedo LF, et al. Role of androgens on MCF-7 breast cancer cell growth and on the inhibitory effect of letrozole. Cancer Res. 2006; 66(15):7775-7782. [PubMed: 16885381]

92. Yamashita H, et al. Predictors of response to exemestane as primary endocrine therapy in estrogen receptor-positive breast cancer. Cancer Sci. 2009; 100(11):2028-2033. [PubMed: 19659610]

93. Khalkhali-Ellis Z, et al. Regulating the tumor suppressor gene maspin in breast cancer cells: a potential mechanism for the anticancer properties of tamoxifen. Clin Cancer Res. 2004; 10(2): 449-454. [PubMed: 14760064]

94. Zhao TP, He GF. A phase II clinical trial of flutamide in the treatment of advanced breast cancer. Tumori. 1988; 74(1):53-56. [PubMed: 3354065]

95. Di Monaco M, et al. Inhibitory effect of hydroxyflutamide plus tamoxifen on oestradiol-induced growth of MCF-7 breast cancer cells. J Cancer Res Clin Oncol. 1995; 121(12):710-714. [PubMed: 7499441] 


\section{Structure of the Human Androgen Receptor - Gene and Protein Organization}

AR gene

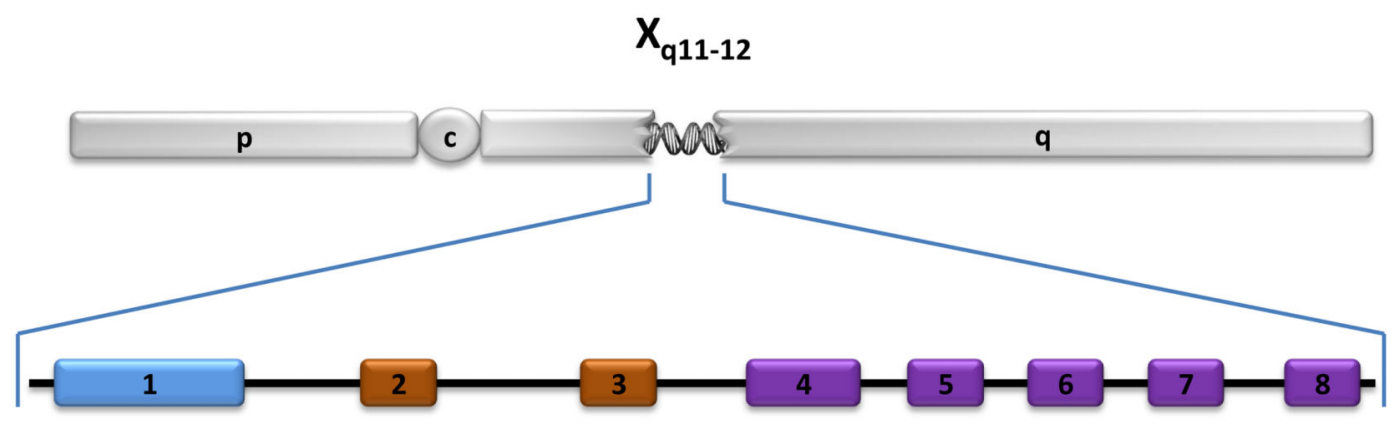

X chromosome

$1 \ldots$

...919

AR protein
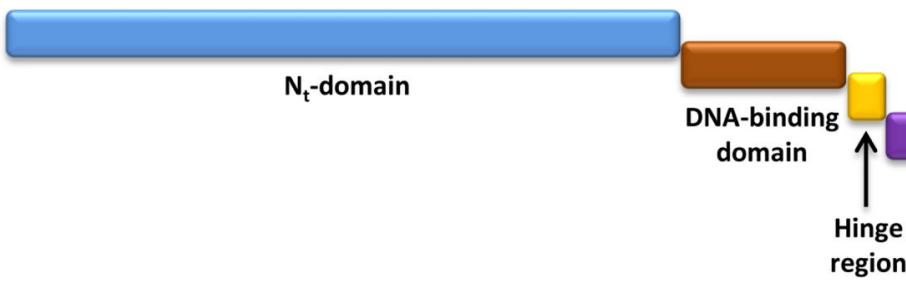

Figure I.

Structure of the Human Androgen Receptor - Gene and Protein Organization 